\title{
EFECTO DE LA SUPLEMENTACIÓN DE CAPSAICINA COMO ESTIMULANTE INMUNOLÓGICO EN POLLOS ROSS
}

\author{
Fernando Sanabria-Naranjo ${ }^{*}$ MVZ, Esp., , Mauricio Mendoza-García, Ing. Agroindustrial, Ph.D.,
}

${ }_{1}$ Facultad de Administración de Empresas Agropecuarias, Universidad Santo Tomás, Colombia

Recibido: 7 de marzo del 2013 Aprobado: 10 mayo del 2013

* Autor de correspondencia: Fernando Sanabria Naranjo, Facultad de Administración de Empresas Agropecuarias, Grupo de investigación Ustagri, Universidad Santo Tomás, Bucaramanga, Colombia, carrera 18 n. ${ }^{\circ}$ 9-27, (57) 76800801 Ext. 1581, correo electrónico: fernandosanabrianaranjo@mail.ustabuca.edu.co

Cómo citar este artículo: Sanabria-Naranjo F, Mendoza-García M. Efecto de la suplementación de capsaicina como estimulante inmunológico en pollos Ross. Spei Domus. 2013; 9(18): 15-27.

Resumen. La capsaicina es una sustancia activa que está presente en el ají picante. En las aves, los capsaicinoides han sido investigados con el fin de prevenir problemas asociados a Salmonella. La tendencia mundial a disminuir los antibióticos como promotores de crecimiento (APC) ha incrementado las investigaciones que busquen reemplazarlos por alternativas naturales como el uso de capsaicinoides. El objetivo de este trabajo fue evaluar el efecto de la adición de capsaicinoides en concentración de 10, 30 y 50 ppm suministrado durante los primeros 16 días de cría en machos y hembras de pollos de engorde de la línea genética Ross. Se evaluó el efecto de la suplementación sobre el sistema inmunológico de las aves de engorde para las enfermedades de bronquitis, Gumboro y Newcastle. Los parámetros zootécnicos medidos fueron: peso vivo, conversión alimenticia, mortalidad, alometría de órganos internos y respuesta inmune. Se observó que ninguna de las aves tratadas rechazara el consumo de alimento que contenía capsaicinoides; por otra parte, no hubo diferencias significativas con respecto a la conversión alimenticia ni al incremento de peso. Los títulos de anticuerpos obtenidos por medio del examen de Elisa contra la enfermedad de Newcastle fueron mayores en las aves vacunadas. Contra las enfermedades de Gumboro y bronquitis, los mayores títulos los presentaron las aves que no recibieron vacunación. Se encontró una tendencia a que los títulos de los anticuerpos se incrementan a medida que se aumenta también la concentración de los capsaicinoides en la dieta de los pollos de engorde.

Palabras clave: aves de engorde, capsaicinoides, Elisa, respuesta inmune.

\section{Effect of Supplementation of Capsaicin as a Natural Stimulant for the Immune System in Ross Broiler Chickens}

Abstract. Capsaicin is the active substance contained in chili peppers. Research has been carried out on capsaicinoids in poultry to prevent salmonella problems. The worldwide trend to reduce antibiotics as growth promoters (AGP's) has led to increased research to replace them with natural alternatives, such as the use of capsaicinoids. The objective of our study was to evaluate the effect of the addition of capsaicinoids in concentrations of 10,30 and $50 \mathrm{ppm}$ administered during the first 16 days of breeding of both male and female Ross broiler chickens. The effect from capsaicinoid supplementation on the immune system of broilers was evaluated with respect to bronchitis, Gumboro and New Castle diseases. The zootechnical parameters measured were body weight, feed conversion, mortality, allometric study of the internal organs and immune response. None of the broilers stopped consuming their feed when it contained capsaicinoids. At the same time, no significant differences were found regarding feed conversion ratio and weight gain. New Castle disease antibody levels found through the Elisa test were higher in the vaccinated broilers; for Gumboro disease and bronchitis, higher antibody levels were found in unvaccinated broilers. We found a tendency for antibody levels to increase as capsaicinoid concentrations were increased in the broilers' diet.

Keywords: broilers, capsaicinoids, Elisa, immune response.
Efeito da suplementação de capsaicina como estimulante imunológico em frangos Ross

Resumo. A capsaicina é uma substância ativa que os "ajís picantes" (um tipo de pimenta) contêm. Nas aves, os capsaicinoides vêm sendo pesquisados com o objetivo de prevenir problemas de salmonela. A tendência mundial para diminuir os antibióticos como promotores de crescimento (APC) vem aumentando as pesquisas que procuram substituí-los por alternativas naturais, por exemplo, mediante o uso de capsaicinoides. $\mathrm{O}$ objetivo de nosso trabalho foi avaliar o efeito da adição de capsaicinoides em concentração de 10, 30 e $50 \mathrm{ppm}$ fornecidos durante os primeiros 16 dias de cria em machos e fêmeas de frangos de corte da linha genética Ross. Avaliou-se o efeito da suplementação sobre o sistema imunológico das aves de corte para as Doenças de Bronquite, Gumboro e Newcastle. Os parâmetros zootécnicos medidos foram: peso vivo, conversão alimentícia, mortalidade, alometria de órgãos internos e resposta imune. Observou-se que nenhuma das aves tratadas rejeitou o consumo de alimento que continha capsaicinoides; por um lado, não houve diferenças significativas a respeito da conversão alimentícia nem do aumento de peso. Os títulos de anticorpos obtidos por meio do Teste de Elisa contra a doença de Newcastle foram maiores nas aves vacinadas. Por outro lado, contra as Doenças de Gumboro e Bronquite, as aves que não receberam vacina apresentaram os maiores títulos. Constamos uma tendência a que os títulos dos anticorpos se aumentaram à medida que se aumenta também a concentração dos capsaicinoides na dieta dos frangos de corte.

Palavras-chave: aves de corte, capsaicinoides, Teste de Elisa, resposta imune. 


\section{Introducción ${ }^{1}$}

Según un nuevo informe de la Dependencia de Estudios de Perspectivas Mundiales de la FAO, la población mundial aumenta a una tasa alarmante y se prevé que para el 2030 habrá más de ocho mil millones de personas en el mundo. Igualmente, se ha incrementado la necesidad de alimentación para este mismo número de personas, por lo que ha repercutido en la búsqueda de soluciones para suplir el déficit de proteína de origen animal. La industria avícola ofrece, debido a su alto grado de industrialización, la oportunidad de obtener alimento de alta calidad a un bajo costo de producción [1].

Las innovaciones y avances tecnológicos en la industria avícola han estado enfocados en la búsqueda de menores conversiones alimenticias, disminuir el uso de antibióticos promotores de crecimiento, el mejoramiento del bienestar animal y, así mismo, la preservación del medio ambiente $[2,3]$.

El consumo de pollo presenta ventajas en comparación con otras fuentes de proteínas de origen animal, ya que brinda proteínas con alto valor biológico; baja cantidad de grasa y purinas; mayor contenido en hierro y ácido fólico, protectores de la anemia; complejo $\mathrm{B}$, que favorece el funcionamiento del sistema nervioso; potasio, magnesio y fósforo, que contribuyen al buen estado neuromuscular en el ser humano. Mientras que la proteína proveniente de la carne de reses es responsable de un mayor trabajo digestivo y mayor contenido en ácidos grasos saturados [2].

En Colombia y otros países se conoce que para el desarrollo industrial de producción de carne de pollo se utilizan antibióticos promotores de crecimiento, y que su utilización ha hecho posible el desarrollo de sistemas intensivos de producción de pollos de engorde, ya que se ha demostrado que contribuyen al mejoramiento del crecimiento y la eficiencia de producción de las aves $[4,5,6]$.

En 1970, la comunidad europea eliminó como promotores aquellos antibióticos que también fueron utilizados en la medicina humana o animal. De este modo, se prohibió en Europa el empleo de tetraciclinas o beta-lactámicos como promotores del crecimiento en el pienso de animales (en Estados Unidos todavía se emplean estos antibióticos) [7]. En la actualidad, en la comunidad europea sólo quedan disponibles como

Este proyecto fue financiado por Colciencias, Ecopetrol, la gobernación de Santander, la Universidad Santo Tomás y Vetécnicos Ltda. promotores cuatro antibióticos (avilamicina, flavofosfolipol, monensina sódica y salinomicina) y su utilización está siendo sometida a una reevaluación [8].

En Estados Unidos actualmente están autorizadas como promotores del crecimiento la penicilina, clortetraciclina, eritromicina, estreptomicina, bacitracina y espectinomicina (todos estos antibióticos son de uso común en la medicina humana), tilosina y virginiamicina (antibióticos con estructuras similares a otros usados en humanos) $[8,9]$.

Hoy en día, ante el uso indiscriminado de antibióticos en la producción animal y con el fin de reducir su uso, se plantean nuevas y diversas alternativas como por ejemplo el uso de aceites esenciales, probióticos (microorganismos vivos que se agregan como suplemento a la dieta), prebióticos (ingredientes no digeribles de la dieta, que estimulan el crecimiento bacteriano en el colon) y simbióticos (son formulaciones que combinan probióticos con prebióticos que actúan sinérgicamente), los cuales representan una alternativa significativa y segura en la producción animal [7].

En Colombia no se tiene conocimiento sobre cuáles y cuántos de estos residuos pueden estar presentes en los productos agropecuarios, ni los productores y asesores técnicos cuentan con información suficiente para evitar el problema de residualidad [10]. Tampoco se dispone de protocolos de diagnóstico ni de información suficientes que permitan cuantificar la problemática con el fin de sentar las bases para su vigilancia y control [11].

El empleo de antibióticos promotores de crecimiento puede traer una acumulación de residuos en la carne o en los huevos, por lo que se han indicado como responsables de la aparición de bacterias resistentes en los humanos a ciertos antibióticos o sus metabolitos [10]. Por esta razón, muchos países, en especial los de la Unión Europea, han dictado medidas restrictivas para su prohibición principalmente para fármacos utilizados como promotores de crecimiento y anticoccidiales. Esta ley en la Comunidad Económica Europea (CEE) entró en vigencia a partir del 1 de enero del 2006 [12], y la tendencia mundial es reproducir esta ordenanza a pesar de las disminuciones en los resultados zootécnicos y económicos en las explotaciones avícolas.

La problemática descrita ha estimulado diferentes procesos de investigación, desarrollo e innovación relacionados con la búsqueda de una serie de productos de origen natural que optimicen la defensa de las aves contra las enfermedades comunes, para ser administrados como aditivos alternativos y poder mantener la 
producción óptima que se obtenía al utilizar los promotores de crecimiento convencionales.

Se denomina al sistema inmune como un conjunto de células, órganos y sustancias que interactúan en defensa del organismo contra agentes extraños [13].

El sistema inmune en las aves corresponde a un conjunto de células que comprende dos tipos de inmunidad: la innata y la de adaptación. La inmunidad innata se puede considerar como el conjunto de herramientas más básicas con que cuenta el organismo para combatir la infección, incluyendo las barreras físicas y químicas, las proteínas de la sangre y las células fagocitarias. La piel, el epitelio de los sistemas respiratorios y digestivos y las secreciones gástricas son ejemplos de las diversas barreras físicas y químicas para evadir a los patógenos [13]. La inmunidad innata se considera como la primera línea de defensa y carece de especificidad, lo cual le permite proteger contra muchos tipos de patógenos. La inmunidad de adaptación se inicia cuando la inmunidad innata no logra detener a algún patógeno invasor y desarrolla el reconocimiento enfocado a las características moleculares específicas del patógeno, dando como resultado una serie de sucesos que eliminan a dicho patógeno y establecen la protección contra desafíos subsiguientes. Esta protección específica puede ser el resultado ya sea de la inmunidad pasiva o de la inmunidad activa [13]. Entre los órganos inmunológicos considerados como primarios podemos encontrar el timo, la bolsa de Fabricio y la médula ósea, mientras que entre los órganos secundarios están el bazo, tejidos linfoides asociados a las mucosas, los nódulos linfáticos y los centros germinales [14].

Entre las principales enfermedades virales existentes en la avicultura industrial están:

- La enfermedad de Newcastle, llamada así por el sitio de origen, descubierta por Doyle en 1926, cuyo agente etiológico es un virus perteneciente a la familia Paramyxoviridae, género Paramixovirus tipo 1 ; es una enfermedad zoonótica y que afecta muchas especies de aves, causando daños irreversibles y pérdidas económicas [15].

- La enfermedad bronquitis infecciosa es infectocontagiosa de alta difusión en las aves, producida por un virus del género Coronavirus $[11,16]$. Se caracteriza por presentar sintomatología respiratoria como tos, estornudos, estertores traqueales, y si afecta el tracto urogenital, puede presentar trastornos en la producción de huevos y daños en los riñones, dependiendo del tipo de cepa que esté atacando $[17,18]$.
- La enfermedad de Gumboro, también conocida como enfermedad infecciosa de la bolsa, es producida por un birnavirus que destruye los linfocitos $\mathrm{B}$ inmaduros de la bolsa de Fabricio en edades tempranas, causando una inmunosupresión [19].

Todas estas enfermedades se intentan controlar mediante la vacunación de las aves, de acuerdo con el metabolismo de anticuerpos maternales que hayan recibido. Se conoce que las vacunas aplicadas en aves pueden desencadenar algún tipo de problema, ya que estas bajan las defensas y los gérmenes oportunistas pueden entrar a atacar a las aves.

Diversas investigaciones buscan una alternativa que pueda presentar sinergia entre las vacunas mediante el uso de agentes naturales, como, por ejemplo, el caso de este estudio suplementando capsaicina. Los capsaicinoides son los compuestos que le dan el sabor picante al ají, son amidas ácidas formadas a partir de vanillilamina y ácidos grasos de 8 a 13 átomos de carbono, conocidos como la capsaicina y la dihidrocapsaicina, que hacen parte del $90 \%$ de los capsaicinoides totales presentes en los ajíes picantes [20].

En los ajíes picantes, el capsaicinoide que se encuentra en mayor cantidad es la capsaicina, que se puede hallar incluso hasta en el $1 \%$ del peso seco del pimiento $[21,22]$. La capsaicina ha sido estudiada en humanos por poseer efectos anticancerígenos [23] y antiinflamatorios [24], desde hace mucho, y es así como en 1919 se describieron los primeros datos de sus efectos biológicos [25, 26]. Los capsaicinoides protegen la mutagénesis bacteriana producida por la aflatoxina B1; así mismo, protegen contra el carcinoma de piel en ratones inducido por el vinil carbamato [27]. También se conocen propiedades antioxidantes que impiden la peroxidación lipídica catalizada por cationes de hierro, y previenen la oxidación del ácido oleico a altas temperaturas [28, 29]. Además, se ha comprobado que previene la oxidación de hidroperóxidos lipídicos provenientes de la oxidación de ácido linóleo [29]. Un inconveniente en su uso en el ser humano es su sabor picante acompañado de sus propiedades molestas al ingerirse, pero este factor no afecta a las aves, pues ellas no tienen problema al ingerir sustancias picantes. Dentro de sus propiedades están las de usarse como analgésico, porque actúa sobre la zona periférica del sistema nervioso, especialmente sobre las neuronas aferentes primarias, reduciendo la trasmisión del impulso nervioso del dolor. Usado en humanos ayuda en enfermedades como la 
artritis reumatoide [30], además de que es antiinflamatorio [24].

En aves se demostró que la administración profiláctica o terapéutica de capsaicina afecta de manera variable la susceptibilidad de los pollos de engorde a la Salmonella [20]. La administración continua de capsaicina en la dieta aumenta la resistencia a la colonización de S. enteritidis y la invasión de órganos en todo el periodo de crecimiento normal sin efectos perjudiciales sobre el crecimiento en pollos de engorde [31,32].

El objetivo de esta investigación fue evaluar el efecto de la adición de diferentes concentraciones de capsaicinoides (10, 30 y $50 \mathrm{ppm}$ ), suministrados en la dieta de pollos de engorde de la línea genética Ross sobre el sistema inmunológico y los resultados médicos y zootécnicos (estudio alométrico).

Se evaluó el efecto de los capsaicinoides sobre los títulos anticuerpos en suero sanguíneo, como respuesta inmunológica a los antígenos aplicados (Newcastle, bornquitis y Gumboro) comparado con las aves no vacunadas. Los diferentes tratamientos fueron suministrados durante los primeros dieciséis días de cría, tanto para aves hembra como para aves macho.

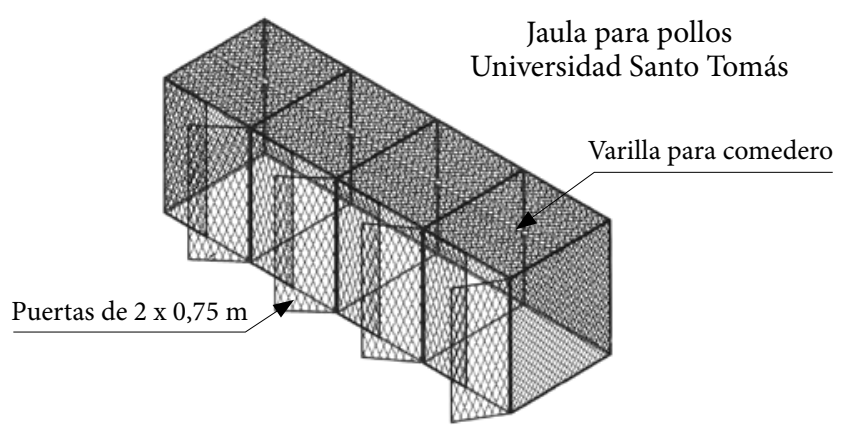

Dimensiones $6 \times 1,75 \times 2 \mathrm{~m}$

Vista isométrica Esc: $1: 25$

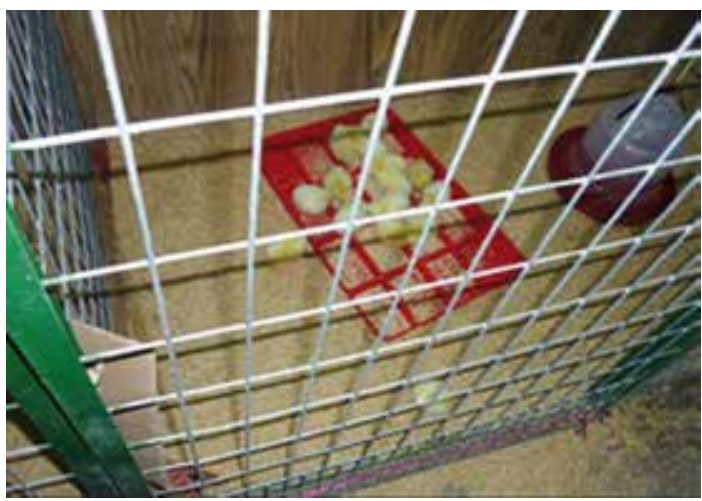

\section{Materiales y métodos}

\section{Ubicación}

La investigación se realizó en la granja experimental El Limonal, propiedad de la Universidad Santo Tomás, localizada en el municipio de Piedecuesta a una altitud de $1.005 \mathrm{msnm}$, con un clima promedio de $24^{\circ} \mathrm{C}$, distante a 17 kilómetros de Bucaramanga.

\section{Preparación de las jaulas experimentales}

Se diseñaron dos jaulas fabricadas de ángulo de hierro y malla galvanizada, las cuales fueron suministradas por industrias Mega Ingeniería Ltda. Cada una de las jaulas medían 6,0 x 1,75 x 2,0 m; estaban divididas en cuatro secciones. Cada sección contaba con una puerta con seguridad de $2,0 \times 0,75 \mathrm{~m}$.

Las jaulas cumplieron con las condiciones de bioseguridad y bienestar animal a una densidad de diez aves por metro cuadrado para la etapa de engorde. En la figura 1 se puede observar el aspecto de las jaulas experimentales utilizadas.
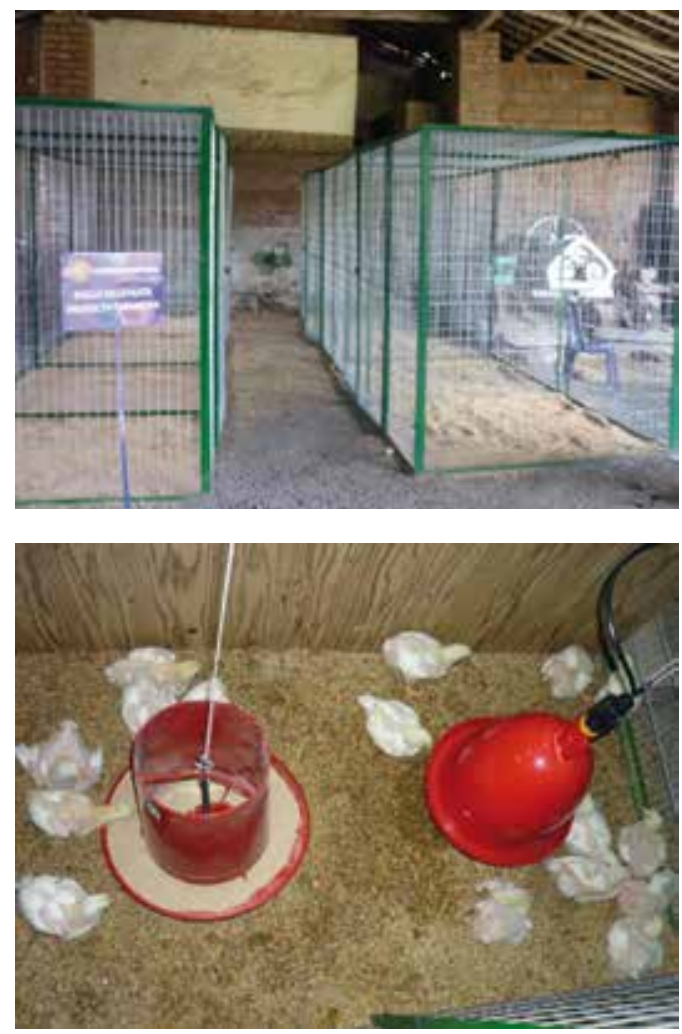

Figura 1. Ilustración de jaulas experimentales utilizadas para la cría y engorde de aves

Fuente: elaboración propia 


\section{Preparación de las aves}

Para este experimento exploratorio se utilizaron 128 aves hembra y 128 aves macho de la línea genética Ross, de un día de edad, suministrados por la empresa Avifonce.

\section{Preparación del alimento}

El alimento fue suministrado por la empresa Nutrimax, Bucaramanga, Colombia. Se realizó un cálculo siguiendo la tabla de recomendación de Aviagen para el consumo de alimento de las aves durante los primeros dieciséis días de la cría.

Para cada una de las secciones experimentales se prepararon 38.690 gramos de alimento balanceado que corresponde al consumo de 64 aves durante la fase experimental.

La concentración de aproximadamente $10 \mathrm{ppm}$ de capsaicinoides se consiguió mezclando 34,73 gramos de ají Capsicumm annuum, variedad bremisculum, deshidratado en 38.930 gramos de alimento.

La concentración de $30 \mathrm{ppm}$ de capsaicinoides se consiguió mezclando 104,19 gramos de ají deshidratado en 38.930 gramos de alimento, y, por último, para obtener 50 ppm de capsaicinoides, se consiguió mezclando 173,6 gramos de ají deshidratado en 38.930 gramos de alimento.

Cada uno de estos lotes de alimento fue rotulado $y$ almacenado en forma independiente en condiciones óptimas de almacenamiento.
Una vez finalizados los dieciséis días de tratamiento con alimento modificado, se continuó suministrando alimento convencional durante los 39 días del experimento.

\section{Diseño experimental}

Se utilizó un diseño experimental de bloques al azar, dividiendo en dos tratamientos el experimento:

T1: aves vacunadas contra la enfermedad de Newcastle, bronquitis infecciosa y Gumboro. La vacunación se realizó por vía ocular, con vacuna de laboratorios Laverlam: cepa La Sota para la enfermedad de Newcastle, cepa Massachusetts para la enfermedad de bronquitis y cepa Lukert para la enfermedad de Gumboro, aplicada directamente en el pico. En la tabla 1 se puede observar el plan de vacunación de las aves experimentales.

T2: aves no vacunadas. Además se estudiaron para cada uno de los tratamientos las diferentes concentraciones de capsaicina: C0, C10, C30 y C50 ppm de capsaicinoides que se suministraron durante los primeros dieciséis días de vida de las aves, continuando hasta terminar su ciclo de 39 días con alimento sin medicar.

En la tabla 2 se puede observar el diseño experimental utilizado.

\section{Conversión alimenticia}

Para calcular la conversión alimenticia de las aves se realizó un pesaje del total del alimento consumido $(\mathrm{kg})$ sobre el peso total de las aves durante cada semana (6)

Tabla 1. Plan de vacunación de las aves experimentales

\begin{tabular}{|c|c|c|c|c|c|c|}
\hline \multirow{2}{*}{ Sexo } & \multirow{2}{*}{ Aves (No) } & \multirow{2}{*}{ Tratamiento } & Antígenos & $\begin{array}{c}\text { Vía de } \\
\text { aplicación }\end{array}$ & Cepa & Tiempo (días) \\
\hline \multirow{2}{*}{ Machos (M) } & \multirow{2}{*}{64} & \multirow{2}{*}{ Vacunado (T1) } & Newcastle & Ocular & La Sota & 10 \\
\cline { 4 - 7 } & & & Bronquitis infecciosa & Ocular & Massachusetts & 10 \\
\cline { 4 - 7 } & & & Gumboro & Oral & Luker Intermedia & 10 \\
\hline
\end{tabular}

Fuente: elaboración propia

Tabla 2. Diseño experimental de aves experimentales

\begin{tabular}{|c|c|c|c|c|}
\hline Sexo & Aves (No) & Tratamiento & Capsaicinoides (ppm) & $\begin{array}{c}\text { Tiempo } \\
\text { (días) }\end{array}$ \\
\hline $\operatorname{Machos}(\mathrm{M})$ & 64 & \multirow{2}{*}{ Vacunado (T1) } & \multirow{4}{*}{$\begin{array}{l}\mathrm{C} 0 \\
\mathrm{C} 10 \\
\mathrm{C} 30 \\
\mathrm{C} 50\end{array}$} & \multirow{4}{*}{16} \\
\hline Hembras $(\mathrm{H})$ & 64 & & & \\
\hline Machos (M) & 64 & \multirow{2}{*}{ No vacunado (T2) } & & \\
\hline $\operatorname{Hembras}(\mathrm{H})$ & 64 & & & \\
\hline
\end{tabular}

Fuente: elaboración propia 
para su evaluación, midiendo los índices alcanzados en cuanto a conversión e incremento de peso. Se utilizó la siguiente fórmula para la obtención de la conversión alimenticia.

Conversión alimenticia $=\frac{\text { Total alimento consumido }(\mathrm{kg})}{\text { Peso total de ave }(\mathrm{kg})}$

\section{Ganancia en peso}

Al finalizar la semana se realizó el pesaje de los animales en forma individual utilizando una báscula digital, y los resultados se anotaron en un registro. Posteriormente se hizo la suma y se dividió por el número de aves pesadas para obtener el promedio de peso por lote.

\section{Diagnóstico médico veterinario de la mortalidad}

Las aves muertas fueron extraidas de las jaulas, numeradas y llevadas al médico veterinario encargado del programa con el fin de realizar la necropsia, en la cual se indicaba el diagnóstico presuntivo de su muerte.

Peso de órganos principales (alometría)

Al finalizar el experimento, se tomaron cinco aves de cada grupo, se sacrificaron para analizar el pesaje de órganos internos como el hígado, la molleja, los intestinos, la bolsa de Fabricio y el timo. Para esto se utilizó una báscula digital de alta precisión y los resultados fueron escritos en una tabla diseñada para el experimento.

\section{Prueba de Elisa para determinación de anticuerpos}

Al finalizar el experimento se tomaron de cada grupo 18 muestras de sangre utilizando una jeringa por cada ave, extrayéndose de la vena alar 2 cc de sangre que fueron depositados en un vial marcado para remitirse al laboratorio Vetécnicos Ltda., el cual realizó el examen de Elisa (Enzyme-Linked Immuno Sorbent Assay), y sus resultados fueron consignados para el análisis estadístico respectivo.

\section{Análisis estadísticos}

Los análisis estadísticos de los resultados se realizaron con el paquete informático Statgraphics Plus 5.1 para
Windows (Manusgístics Inc., Rockville, MD, USA), mediante análisis de la varianza (Anova) simple y multifactorial, para evaluar el efecto causado por uno o más tratamientos durante el estudio. Las diferencias mínimas entre las medias se establecieron mediante intervalos LSD (Fisher) y contraste múltiple de rangos con un nivel de confianza al $90 \mathrm{y}$ al $95 \%$.

\section{Resultados y discusión}

Es importante enfatizar que ningún ave del experimento presentó rechazo a las diferentes mezclas de alimento en relación con las concentraciones de capsaicinoides utilizadas.

No hubo diferencia significativa en cuanto al incremento de peso de las hembras tanto vacunadas como no vacunadas. Sin embargo, respecto a la concentración de los capsaicinoides, sí se presentaron diferencias significativas a un nivel de confianza del $95 \%$ mediante el procedimiento de las menores diferencias significativas de Fisher (LSD), por medio del cual se encontró que los animales que fueron alimentados con capsaicina a $10 \mathrm{ppm}$ obtuvieron un mayor aumento de peso con un valor promedio de $1.896,72$ gramos a los 39 días. Asimismo, se pudo comprobar que las hembras que obtuvieron el menor peso fueron las que consumieron $50 \mathrm{ppm}$ con un valor promedio de 1.784,16 gramos.

En la tabla 3 se pueden observar los pesos y sus diferencias tanto para machos como para hembras, según tratamiento y concentración de capsaicinoides. Las letras mayúsculas por cada columna significan diferencias estadísticamente significativas respecto del tratamiento T1 vs T2. Las letras minúsculas en cada columna significan diferencias significativas respecto a la concentración de capsaicina.

Tabla 3. Peso de las aves experimentales para el día 39 de vida

\begin{tabular}{|c|c|c|c|}
\hline Tratamiento & $\begin{array}{c}\text { Concentración } \\
\text { capsaicinoides }\end{array}$ & $\begin{array}{c}\text { Peso aves } \\
\text { machos (g) }\end{array}$ & $\begin{array}{c}\text { Peso aves } \\
\text { hembras (g) }\end{array}$ \\
\hline $\mathrm{T} 1$ & $\mathrm{C} 0$ & $1.885 \pm 198 \mathrm{Bab}$ & $1.844 \pm 239 \mathrm{~A}$ \\
\hline $\mathrm{T} 1$ & $\mathrm{C} 10$ & $1.777 \pm 165 \mathrm{Ba}$ & $1.853 \pm 154 \mathrm{~A}$ \\
\hline $\mathrm{T} 1$ & $\mathrm{C} 30$ & $1.847 \pm 208 \mathrm{Bab}$ & $1.782 \pm 446 \mathrm{~A}$ \\
\hline $\mathrm{T} 1$ & $\mathrm{C} 50$ & $1.806 \pm 223 \mathrm{Bbc}$ & $1.808 \pm 301 \mathrm{~A}$ \\
\hline $\mathrm{T} 2$ & $\mathrm{C} 0$ & $1.733 \pm 154 \mathrm{Aab}$ & $1.879 \pm 212 \mathrm{~A}$ \\
\hline $\mathrm{T} 2$ & $\mathrm{C} 10$ & $1.894 \pm 222 \mathrm{Aa}$ & $1.938 \pm 179 \mathrm{~A}$ \\
\hline $\mathrm{T} 2$ & $\mathrm{C} 30$ & $1.784 \pm 127 \mathrm{Aab}$ & $1.796 \pm 193 \mathrm{~A}$ \\
\hline $\mathrm{T} 2$ & $\mathrm{C} 50$ & $1.740 \pm 273 \mathrm{Abc}$ & $1.762 \pm 191 \mathrm{~A}$ \\
\hline
\end{tabular}

Fuente: elaboración propia 
La posible diferencia de peso entre las aves vacunadas y las no vacunadas fue debido al estrés ocasionado por la manipulación y los efectos de los antígenos suministrados. Se puede observar que la media del peso en las aves vacunadas y alimentadas con $50 \mathrm{ppm}$ disminuyó drásticamente, debido a la muerte de un ave en el último día, y la causa fue diagnosticada como infarto cardiaco.

En cuanto a las aves macho y el efecto de tratamiento se encontraron diferencias significativas respecto al tratamiento, en el que las aves sin vacuna (T2) presentaron un peso promedio de 1.859,5 gramos respecto a las aves con vacuna (T1) con un peso promedio de 1.790 gramos. Esto debido posiblemente a que las aves vacunadas (T2) presentaron un estrés por el manejo y por la acción de los antígenos aplicados.

En relación con el efecto de la concentración de capsaicinoides sobre la evolución de peso en aves macho, se encontraron diferencias significativas. Los animales alimentados con capsaicinoides a $10 \mathrm{ppm}$ fueron los que tuvieron un mayor aumento de peso con un valor promedio de 1.900,1 gramos. Los que menor ganancia de peso obtuvieron fueron las aves macho alimentadas con $50 \mathrm{ppm}$ de capsaicinoides, con un valor promedio de 1.772,5 gramos, como se puede observar en la tabla 2.

Aparentemente en el experimento se puede concluir que la dosis que dio mejor resultado en cuanto a la ganancia de peso, tanto en machos como en hembras, fue la de las aves que consumieron los capsaicinoides a $10 \mathrm{ppm}$. La evolución de peso de las aves macho con vacuna y sin esta se puede observar en la figura 2.

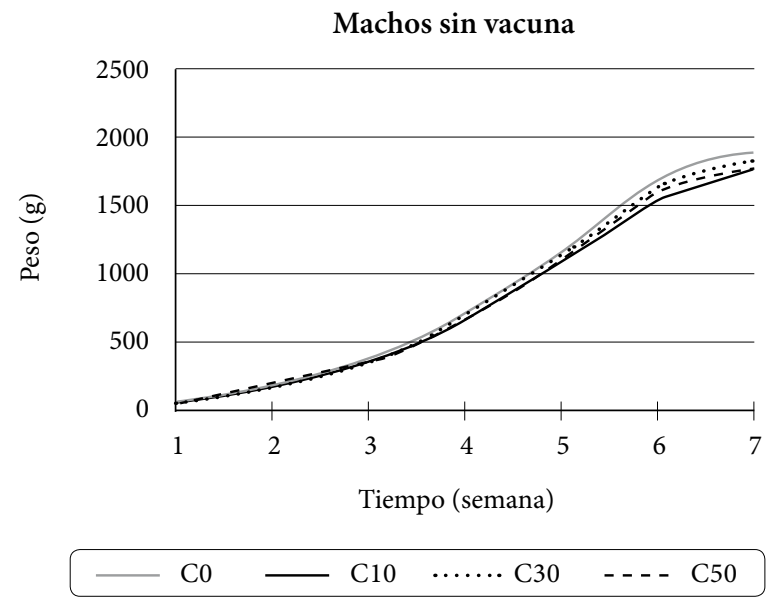

En relación con la conversión alimenticia, los animales no presentaron diferencias estadísticamente significativas, como se puede encontrar en la figura 3.

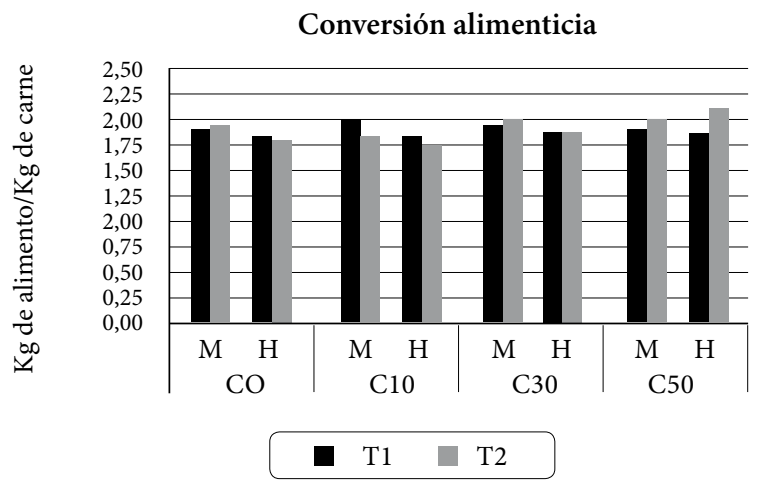

Figura 3. Conversión de alimento de las aves experimentales Fuente: elaboración propia

En cuanto a la mortalidad ocurrida durante el experimento, se puede observar en la tabla 4 .

Tabla 4. Mortalidad de las aves experimentales

\begin{tabular}{|c|c|c|c|c|c|c|c|c|}
\hline \multirow{2}{*}{ Semana } & \multicolumn{2}{|c|}{ CO } & \multicolumn{2}{c|}{ C10 } & \multicolumn{2}{c|}{ C30 } & \multicolumn{2}{c|}{ C50 } \\
\cline { 2 - 9 } & T1 & T2 & T1 & T2 & T1 & T2 & T1 & T2 \\
\hline 1 & 0 & 3 & 1 & 2 & 0 & 1 & 0 & 0 \\
\hline 2 & 0 & 0 & 0 & 1 & 0 & 0 & 0 & 0 \\
\hline 3 & 0 & 0 & 0 & 0 & 0 & 0 & 0 & 0 \\
\hline 4 & 0 & 0 & 0 & 0 & 0 & 0 & 0 & 0 \\
\hline 5 & 0 & 0 & 0 & 1 & 0 & 0 & 0 & 0 \\
\hline 6 & 0 & 0 & 0 & 0 & 0 & 0 & 1 & 0 \\
\hline Total & 0 & 3 & 1 & 4 & 0 & 1 & 1 & 0 \\
\hline
\end{tabular}

Fuente: elaboración propia

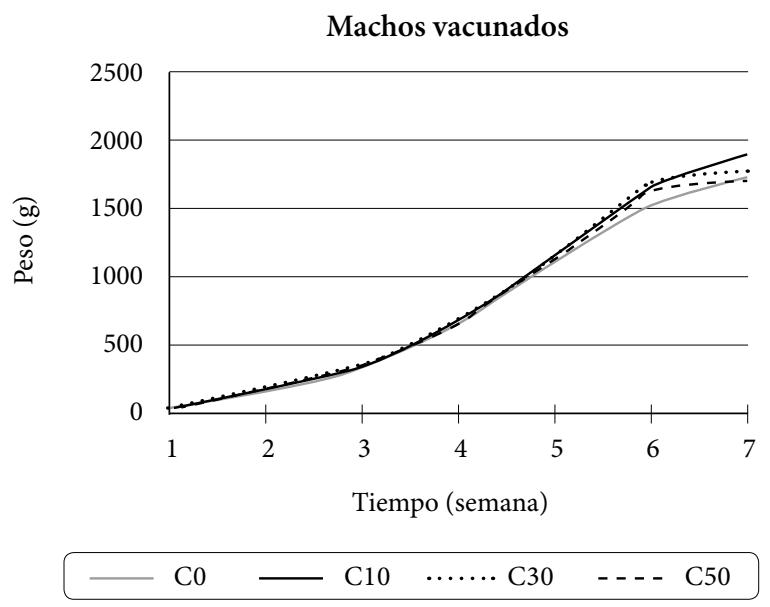

Figura 2. Incremento de peso durante 39 días de aves macho en experimento con vacuna y sin esta, alimentados a diferentes concentraciones de capsaicinoides Fuente: elaboración propia 
Durante todo el experimento se presentó una mortalidad de 10 aves en total, lo que llevó a obtener un porcentaje del 3,9\%. De esta mortalidad el mayor número ocurrió durante la primera semana de vida (7 aves). También se puede observar que, independientemente de la concentración de capsaicinoides, el tratamiento T2 (aves sin vacuna) presentó el mayor número de aves muertas ( 7 aves), mientras que el tratamiento T1 presentó el menor número de aves muertas.

En la tabla 5 se puede observar el número de aves muertas y su correspondiente diagnóstico veterinario. La causa probable de la muerte se debe a fallas en el proceso de incubación y se concluye que las diferentes concentraciones de capsaicinoides no tuvieron nada que ver con esta mortalidad.

En relación con el estudio alométrico de los diferentes órganos de las aves experimentales, no se encontraron diferencias significativas en bazo, bolsa de Fabricio y timo, como se evidencia en la tabla 6 .

Para el caso del hígado se pueden observar los valores medios del efecto del tratamiento con vacuna y sin esta sobre el peso del órgano de las aves donde muestran diferencias estadísticamente significativas respecto a su peso, en las que T1 (aves vacunadas) son las que presentan un hígado más pesado.

Al comparar las diferentes concentraciones de capsaicinoides usados en las dietas y al evaluar el peso del hígado podemos encontrar que existe una diferencia significativa, en la que el grupo de aves que consumieron $30 \mathrm{ppm}$ (C30) son las que obtuvieron el mayor peso.

Respecto al peso de intestino, se encontraron diferencias significativas, un mayor peso en las aves que fueron vacunadas.

Al comparar las diferentes concentraciones de capsaicinoides usados en la dietas, podemos encontrar que existió una diferencia significativa entre el grupo de aves alimentadas con C10, lo que llevó a obtener el mejor peso en relación con el peso del intestino de los otros tratamientos. Para el caso de la evaluación de anticuerpos por el método Elisa contra la enfermedad Newcastle, hubo diferencias estadísticamente significativas, tanto para aves hembra como para aves macho respecto a los tratamientos T1 y T2, como se ve en la figura 4, en la que los títulos mayores son para las aves vacunadas.

Tabla 5. Descripción de las aves muertas durante el experimento

\begin{tabular}{|c|c|c|c|c|}
\hline Tratamiento (Tt0) & Concentraciones & Sexo & Diagnóstico & Edad muerte (días) \\
\hline T2 & C0 & Macho & Nefritis y deshidratación & 1 \\
\hline T2 & C10 & Macho & Nefritis y deshidratación & 1 \\
\hline T1 & C10 & Macho & Onfalitis & Onfalitis \\
\hline T2 & CO & Macho & Onfalitis & 3 \\
\hline T2 & CO & Macho & Onfalitis & 3 \\
\hline T2 & C10 & Hembra & Septicemia & 3 \\
\hline T2 & C30 & Macho & Infarto & 9 \\
\hline T2 & C10 & Hembra & Infarto de miocardio & 34 \\
\hline T2 & C10 & Macho & & 39 \\
\hline T1 & C50 & Hembra & & \\
\hline
\end{tabular}

Fuente: elaboración propia

Tabla 6. Efecto del tratamiento y concentración de capsaicinoides sobre el peso de órganos relevantes en el engorde de pollos

\begin{tabular}{|c|c|c|c|c|c|c|c|}
\hline Tto & Capsaicina & Hígado $(\mathrm{g})$ & Bazo (g) & Molleja (g) & Intestino (g) & Bolsa Fabricio (g) & Timo $(\mathrm{g})$ \\
\hline $\mathrm{T} 1$ & C0 & $37,9 \pm 8.0$ & $2,1 \pm 0.7$ & $44,8 \pm 8,9$ & $58,9 \pm 7,3$ & $1,8 \pm 0,1$ & $3,1 \pm 0,9$ \\
\hline $\mathrm{T} 1$ & $\mathrm{C} 10$ & $44,1 \pm 5,6$ & $2,2 \pm 1,2$ & $50,7 \pm 12,2$ & $88,0 \pm 5,5$ & $2,4 \pm 1,3$ & $2,7 \pm 0,5$ \\
\hline $\mathrm{T} 1$ & C30 & $47,5 \pm 1,8$ & $3,4 \pm 0,5$ & $58,3 \pm 1,0$ & $68,7 \pm 10,7$ & $3,4 \pm 0,7$ & $3,5 \pm 0,3$ \\
\hline $\mathrm{T} 1$ & C50 & $44,2 \pm 2,6$ & $2,0 \pm 0,0$ & $40,8 \pm 7,4$ & $71,9 \pm 11,7$ & $2,1 \pm 0,1$ & $3,5 \pm 0,1$ \\
\hline $\mathrm{T} 2$ & $\mathrm{C} 0$ & $28,6 \pm 1,1$ & $1,6 \pm 0,4$ & $39,9 \pm 0,5$ & $56,5 \pm 4,3$ & $2,4 \pm 0,6$ & $3,5 \pm 0,5$ \\
\hline $\mathrm{T} 2$ & $\mathrm{C} 10$ & $35,5 \pm 3,4$ & $1,6 \pm 0,6$ & $53,9 \pm 11,1$ & $66,0 \pm 16,8$ & $2,4 \pm 0,3$ & $2,7 \pm 0,3$ \\
\hline $\mathrm{T} 2$ & C30 & $40,4 \pm 0,8$ & $2,4 \pm 1,6$ & $43,4 \pm 6,9$ & $63,2 \pm 10,8$ & $2,1 \pm 0,0$ & $3,3 \pm 0,2$ \\
\hline $\mathrm{T} 2$ & C50 & $32,9 \pm 5,8$ & $1,8 \pm 0,2$ & $37,0 \pm 7,1$ & $54,5 \pm 5,3$ & $1,9 \pm 0,6$ & $3,0 \pm 0,5$ \\
\hline
\end{tabular}

Fuente: elaboración propia 
Medias y porcentajes de intervalos LSD (95,0\%)

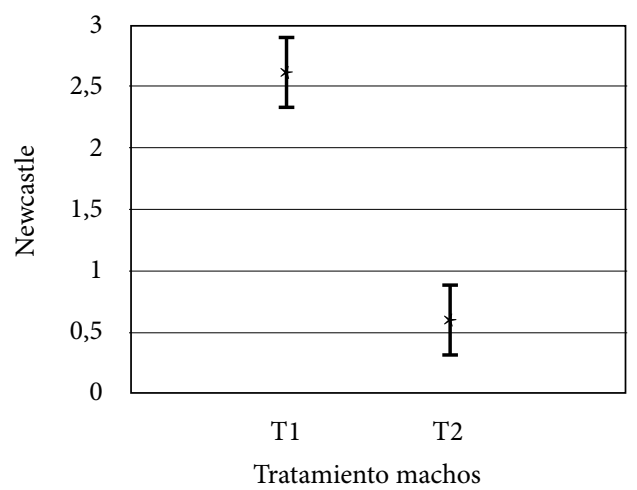

Medias y porcentajes de intervalos LSD $(95,0 \%)$

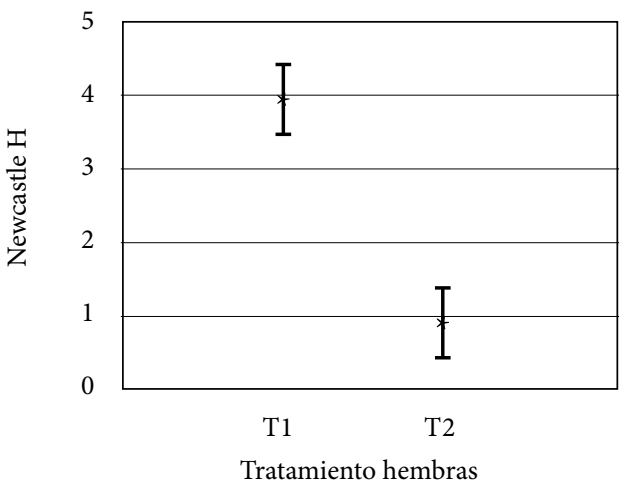

Figura 4. Comparación de medias de los títulos del examen Elisa para la enfermedad del Newcastle tanto para aves macho como para aves hembra

Fuente: elaboración propia

Respecto al efecto de las diferentes concentraciones de capsaicinoides suministrados a las hembras en la dieta para los títulos del examen Elisa de la enfermedad del Newcastle, se pudo observar que al aumentar la concentración de capsaicinoides aumentan los títulos de los anticuerpos. Esto va en concordancia con otros autores como los investigadores del Departamento de Bioquímica y Biología Molecular de la Escuela de Medicina de la Universidad de Alcalá, Madrid, quienes demostraron que en humanos la capsaicina indujo un aumento en la viabilidad celular de las células LNCaP de cáncer de próstata, sensibles a andrógeno.

En la tabla 7 se pudo observar el contraste múltiple de rangos según la concentración de capsaici-

Tabla 7. Contraste múltiple de rangos para aves hembra vacunadas contra Newcastle según concentración de capsaicinoides

\begin{tabular}{|c|c|c|c|c|}
\hline Capsaicina & Recuento & $\begin{array}{c}\text { LSD } \\
\text { Media LS }\end{array}$ & Sigma LS & $\begin{array}{c}\text { Grupos } \\
\text { homogéneos }\end{array}$ \\
\hline $\mathrm{C} 0$ & 27 & $1.907,4$ & 510,418 & $\mathrm{X}$ \\
\hline $\mathrm{C} 10$ & 27 & $1.998,7$ & 508,349 & $\mathrm{X}$ \\
\hline $\mathrm{C} 30$ & 27 & $2.270,85$ & 508,348 & $\mathrm{XX}$ \\
\hline C50 & 29 & $3.285,52$ & 490,501 & $\mathrm{X}$ \\
\hline \multicolumn{3}{|l|}{ Contraste } & Diferencias & +/- Límites \\
\hline \multicolumn{3}{|l|}{$\mathrm{C} 0-\mathrm{C} 10$} & $-91,303$ & $1.194,46$ \\
\hline \multicolumn{3}{|l|}{$\mathrm{C} 0-\mathrm{C} 30$} & $-363,451$ & $1.194,45$ \\
\hline \multicolumn{3}{|l|}{$\mathrm{C} 0-\mathrm{C} 50$} & ${ }^{\star} .1378,12$ & $1.175,71$ \\
\hline \multicolumn{3}{|l|}{ C10-C30 } & $-272,148$ & $1.192,83$ \\
\hline \multicolumn{3}{|l|}{ C10-C50 } & ${ }^{*}-1.286,82$ & $1.172,47$ \\
\hline \multicolumn{3}{|l|}{ C30-C50 } & $-1.014,67$ & $1.172,47$ \\
\hline
\end{tabular}

${ }^{*}$ Indica una diferencia significativa

Fuente: elaboración propia noides suministrado en la dieta de las hembras. Se observaron claramente dos grupos ( $\mathrm{C} 0, \mathrm{C} 10$ y C30) que son homogéneos y presentan diferencias entre C30 y C50.

Sorprendentemente, este comportamiento no fue similar para los machos (ver tabla 8), en los que los títulos de anticuerpos disminuyeron conforme aumentaba la concentración de capsaicinoides.

Para el caso de los títulos para la enfermedad de bronquitis infecciosa, en la figura 5 se puede observar que hubo diferencias estadísticamente significativas, tanto para hembras como para machos, respecto a los tratamientos $\mathrm{T} 1$ y T2, en las que las aves no vacunadas (T2) son las que presentaron mayores títulos.

Tabla 8. Contraste múltiple de rangos para aves macho vacunadas contra Newcastle según concentración de capsaicinoides

\begin{tabular}{|c|c|c|c|c|}
\hline Capsaicina & Recuento & $\begin{array}{c}\text { LSD } \\
\text { Media LS }\end{array}$ & Sigma LS & $\begin{array}{c}\text { Grupos } \\
\text { homogéneos }\end{array}$ \\
\hline C30 & 32 & $1.117,81$ & 304,344 & $\mathrm{X}$ \\
\hline C50 & 30 & $1.416,67$ & 314,325 & $\mathrm{XX}$ \\
\hline $\mathrm{C} 0$ & 28 & $1.416,67$ & 326,191 & XX \\
\hline $\mathrm{C} 10$ & 30 & $2.034,79$ & 315,026 & $\mathrm{X}$ \\
\hline \multicolumn{3}{|l|}{ Contraste } & Diferencias & +/- Límites \\
\hline \multicolumn{3}{|l|}{$\mathrm{C} 0-\mathrm{C} 10$} & $-299,081$ & 898,51 \\
\hline \multicolumn{3}{|l|}{$\mathrm{C} 0-\mathrm{C} 30$} & 617,893 & 883,938 \\
\hline \multicolumn{3}{|l|}{$\mathrm{C} 0-\mathrm{C} 50$} & 319,038 & 897,545 \\
\hline \multicolumn{3}{|l|}{ C10-C30 } & *916,9738 & 867,894 \\
\hline \multicolumn{3}{|l|}{ C10-C50 } & 618,119 & 881,75 \\
\hline \multicolumn{3}{|l|}{ C30-C50 } & $-298,854$ & 866,896 \\
\hline
\end{tabular}

${ }^{*}$ Indica una diferencia significativa

Fuente: elaboración propia 

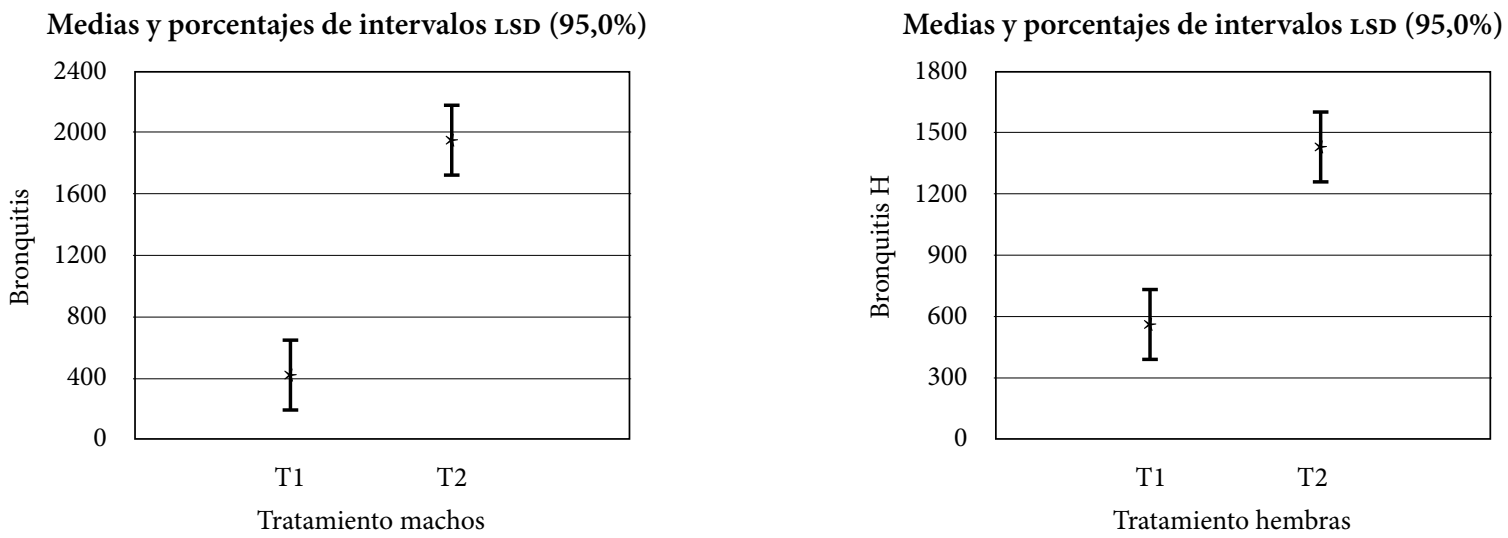

Figura 5. Comparación de medias de los títulos del examen Elisa para la enfermedad de bronquitis tanto para machos como para hembras

Fuente: elaboración propia

Respecto al efecto de las diferentes concentraciones de capsaicinoides suministrados a los machos en la dieta para los títulos del examen de Elisa de la enfermedad de bronquitis infecciosa, en la figura 6 se puede observar que al aumentar la concentración de capsaicinoides aumentan los títulos de los anticuerpos.

Para el caso de la evaluación de anticuerpos contra la enfermedad de Gumboro o enfermedad infecciosa de la bolsa de Fabricio, se pudo observar en la figura 7 que hubo diferencias estadísticamente significativas, tanto para hembras como para machos, respecto a los tratamientos T1 y T2, en las que los títulos más altos son para las aves que no recibieron vacuna.

Respecto al efecto de las diferentes concentraciones de capsaicinoides suministrados a las hembras en la dieta para los títulos del examen Elisa de la enfermedad de Gumboro, se pudo observar que no existió

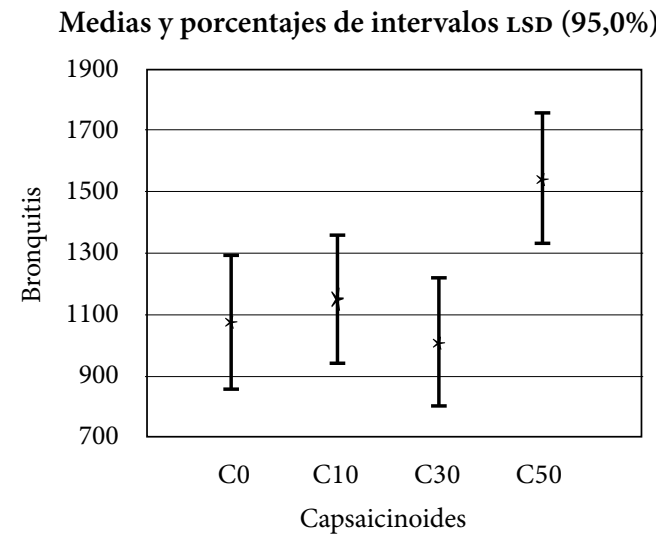

diferencia significativa en relación con las diversas concentraciones aplicadas.

Para las aves macho, en cuanto al suministro de las diferentes concentraciones de capsaicinoides, las que no consumieron capsaicinoides presentaron mayores títulos a la enfermedad de Gumboro. Aparentemente existe un antagonismo entre alimentar con capsaicinoides a las aves macho con el descenso de los títulos de la enfermedad de Gumboro.

\section{Discusión y conclusiones}

Es muy importante enfatizar que ningún ave del experimento presentó rechazo a las diferentes mezclas de alimento en relación con concentraciones de capsaicinoides.

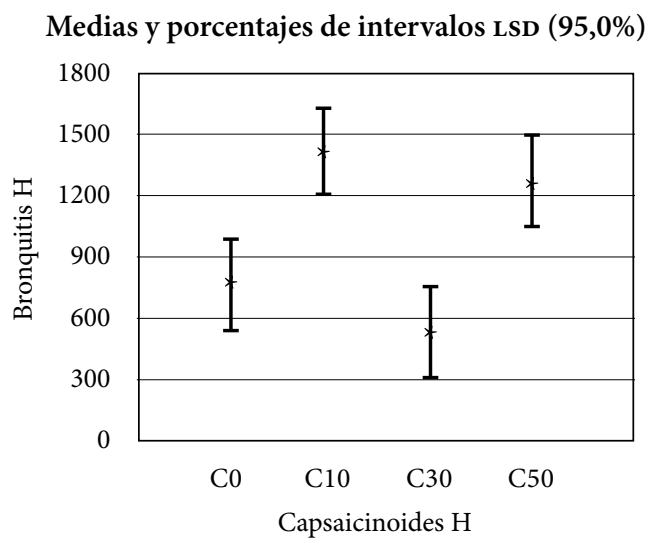

Figura 6. Comparación de medias de los títulos del examen Elisa para la enfermedad bronquitis en relación con la concentración de capsaicinoides suministrados en la dieta de las aves macho y las aves hembra $(\mathrm{H})$

Fuente: elaboración propia 

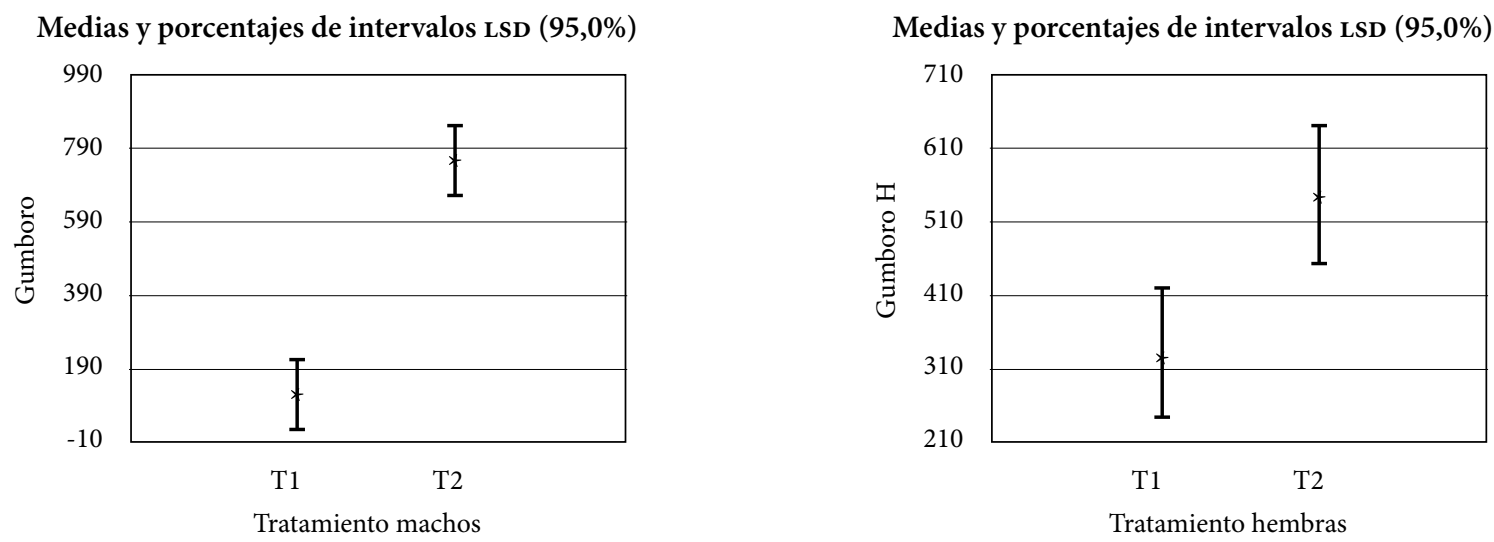

Figura 7 Comparación de medias de los títulos del examen Elisa para la enfermedad de Gumboro tanto para aves macho como para aves hembra

Fuente: elaboración propia

Las aves experimentales no presentaron diferencia significativa entre el T1 y T2, con respecto al incremento de peso.

Aunque no hubo diferencias significativas en cuanto al incremento de peso, la mejor respuesta correspondió al tratamiento en el que las aves Ross macho y hembra consumieron $10 \mathrm{ppm}$, y el resultado de menor peso lo obtuvieron las aves hembra que consumieron $50 \mathrm{ppm}$ de capsaicinoides. A partir de esto se pudo recomendar la adición de 10 ppm en la dieta alimentaria.

Con respecto al incremento de peso, se puede afirmar que no hay diferencia significativa entre las aves vacunadas y las no vacunadas, lo que lleva a concluir que la aplicación de antígenos no influye en este parámetro.

La conversión alimenticia de ambos lotes experimentales no presentó diferencias significativas.

La mortalidad del lote en general estuvo dentro de los parámetros normales con un promedio del 3,9\%. El mayor número de muertos (7 de 10) se presentó en la primera semana de vida y el diagnóstico obtenido fue onfalitis y deshidratación, que indica que los capsaicinoides no incidieron sobre ella. Igualmente, el porcentaje fue mayor en aves T2 (no vacunadas) comparadas con las T1 (vacunadas).

En cuanto al peso del hígado, el mayor peso se encontró en el lote T1 vacunado comparado con el lote $\mathrm{T} 2$ sin vacuna. Y el mayor peso se encontró en aves que consumieron $30 \mathrm{ppm}$.

Los intestinos más pesados se encontraron en el lote de aves que recibieron vacuna (T1), comparados con las aves del lote T2 que no recibieron vacunación. Igualmente, las que presentaron el mejor peso fueron las aves que consumieron $10 \mathrm{ppm}$.
En cuanto a la molleja, timo y bolsa de Fabricio no hubo diferencia significativa entre los dos tratamientos.

De acuerdo con los resultados obtenidos en los análisis de sangre por la prueba Elisa se concluye que: para la enfermedad de Newcastle, las aves que presentaron un mayor título corresponden tanto a machos como a hembras del lote $\mathrm{T} 1$ (vacunados) contra las aves del lote T2 que no recibieron vacuna. Al aumentarse la concentración de capsaicinoides, también se encontró un aumento de los títulos contra esta enfermedad en las hembras, pero caso contrario se obtuvo en los machos, en los cuales los títulos disminuyeron (efecto antagónico).

Para la enfermedad de bronquitis infecciosa los mayores títulos se obtuvieron en las aves que no recibieron vacunación (T2) tanto en hembras como en machos. En los machos, al aumentar la concentración de capsaicinoides también se vio un incremento en los títulos. En las hembras, al suministrarse diferentes concentraciones de capsaicinoides, se puede observar que hay una homogeneidad entre las aves testigo y el grupo que consumió $30 \mathrm{ppm}$; y una diferencia significativa entre los grupos que consumieron $30 \mathrm{ppm}$ y los que consumieron $50 \mathrm{ppm}$.

Para la enfermedad de Gumboro, se presentaron diferencias significativas entre machos y hembras del lote no vacunado $\mathrm{T} 2$; se observaron títulos más altos para las aves que no recibieron la vacuna, y no se presentaron diferencias significativas de acuerdo con la concentración de capsaicina en hembras. Los machos testigos que no consumieron capsaicina fueron los que presentaron títulos más altos. Igualmente, se observa antagonismo entre el alimento con capsaicinoides en los machos con el descenso de sus títulos. 
Según este estudio, se recomienda adicionar capsaiconoides a la dieta de pollos de engorde durante la fase de crecimiento, como sustituto de promotores de crecimiento, con el fin de estimular el potencial fisiológico de los intestinos, logrando así tener una mejor absorción de los alimentos y estimulando los órganos inmunológicos que se encuentran en ellos.

\section{Referencias}

[1] FAO. Alimentos y población: la FAO anticipa. 2000 [Consultado: 14 de febrero del 2013]. Disponible en: http:// www.fao.org/NOTICIAS/2000/000704-s.htm.

[2] Torres C, Zarazaga, M. Antibióticos como promotores del crecimiento en animales: ¿Vamos por el buen camino? Gac Sanit. Mar-Abr 2002; 16(2): 109-12.

[3] Díaz R. Calidad e inocuidad de los alimentos. Proteína animal: un desafío en producción animal tradicional. Santa Fe (Argentina): El Cid Editor; 2009. p. 29.

[4] Peterson RA, Carpenter GH, Jones WT, Kula JA. Research note: effect of tetracycline hydrochloride and oxytetracycline hydrochloride given via drinking water on early mortality of broiler chicks from twenty-eightweek-old dams. Poult Sci. Abr 1991; 70(4): 1040-2.

[5] Rosen GD. Antibacterials in poultry and pig nutrition. En: Wallace RJ, Chesson A, editores. Biotechnology in Animal Feeds and Feeding. Weinheim: vCH; 1995. p. 143-72.

[6] Hernández F, Madrid J, García V, Orengo J, Megías MD. Influence of two plant extracts on broilers performance, digestibility, and digestive organ size. Poult Sci. Feb 2004; 83(2): 169-74.

[7] Castro M, Rodríguez F. Levaduras: probióticos y prebióticos que mejoran la producción animal. Revista Corpoica. 2005; 6(1): 26.

[8] European Federation of Animal Health. Antibiotics for Animals: A Fedesa perspective on antibiotics, animal health and the resistance debate. Bruselas: Fedesa. 1999: 17-22.

[9] Lin J, Hunkapiller AA, Layton AC, Chang YJ, Robbins KR. Response of intestinal microbiota to antibiotic growth promoters in Chickens. Foodborne Pathog Dis. 2013; 10(4): 331-7.

[10] Dildo ML. Residuos químicos en alimentos de origen animal: problemas y desafíos para la inocuidad alimentaria en Colombia. Revista Corpoica-Ciencia y Tecnología Agropecuaria. 2008; 9(1): 124.

[11] Álvarez DCM, Jaimes-Olaya JA, Correa JJ, Nieto GCR, Alfonso VJV. Persistencia del virus vacunal de bronquitis infecciosa en una granja avícola de pollos de engorde en Colombia. Rev Cient (Maracaibo). 2012; 22(2): 120-7.

[12] Comunidad Económica Europea. Directiva 18/31. 2003.

[13] Davison TF, Kaspers B, Schat KA. Avian immunology. Amsterdam; Boston; London: Elsevier/Academic Press; 2008; Disponible en: http://site.ebrary.com/ id/10209712.

[14] Davinson F, Kaspers B, Karel AS. Avian Inmunology. Press A, editor. San Diego, CA, 2008.

[15] Rahman MS, Rabbani MG, Uddin MJ, Chakrabartty A, Her M. Prevalence of Avian Influenza and Newcastle Disease Viruses in poultry in selected areas of Bangladesh using rapid antigen detection kit. Arch Clin $\mathrm{Mi}$ crobiol. 2012; 3(1).

[16] Landman WJM, Matthijs MGR, van Eck JHH. Effect of anti-inflammatory drugs on colibacillosis lesions in broilers after Infectious Bronchitis Virus and subsequent Escherichia coli infection. Vet Q. 2012; 32(1): 25-9.

[17] Gelb Jr J, Ladman BS, Pope CR, Ruano JM, Brannick EM, Bautista DA et al. Characterization of nephropathogenic infectious bronchitis virus DMv/1639/11 recovered from Delmarva broiler chickens in 2011. Avian Dis. Mar 2013; 57(1): 65-70.

[18] Almeida DO, Tortelly R, Nascimento ER, Chagas MA, Khan MI, Pereira VLA. Avian infectious bronchitis and deep pectoral myopathy-A case control study. Poult Sci. Dic 2012; 91(12): 3052-6.

[19] Boudaoud A, Alloui N. Evaluation of the safety of live attenuated vaccine viruses against infectious bursal disease (Gumboro disease) in conventional broiler chicks. Rev Sci Tech. Dic 2008; 27(3): 793-802.

[20] Orndorff BW, Novak CL, Pierson FW, Caldwell DJ, McElroy AP. Comparison of prophylactic or therapeutic dietary administration of capsaicin for reduction of Salmonella in broiler chickens. Avian Dis. Dic 2005; 49(4): 527-33.

[21] Bennett DJ, Kirby GW. Constitution and biosynthesis of capsaicin. J Chem Soc C. 1968: 442-6.

[22] Ham H, Sung M, Kim Y, Choi Y, Jeong HS, Lee J. Determination of capsaicinoids in selected commercial pepper powders and pepper-containing products using HPLC and method validation. J Korean Soc Food Sci Nutr. 2012; 41(6): 870-4.

[23] Unnikrishnan MC, Kuttan R. Tumour reducing and anticarcinogenic activity of selected spices. Cancer Lett. May 1990; 51(1): 85-9.

[24] Kim CS, Kawada T, Kim BS, Han IS, Choe SY, Kurata $\mathrm{T}$ et al. Capsaicin exhibits anti-inflammatory property 
by inhibiting IkB-a degradation in LPS-stimulated peritoneal macrophages. Cell Signal. Mar 2003; 15(3): 299-306.

[25] Nelson EK. The constitution of capsaicin, the pungent principle of capsicum. II. J Am Chem Soc. 1920; 42(3): 597-9.

[26] Nelson EK, Dawson LE. The constitution of capsaicin, the pungent principle of capsicum. III. J Am Chem Soc. 1923; 45(9): 2179-81.

[27] Surh YJ, Lee RCJ, Park KK, Mayne ST, Liem A, Miller JA. Chemoprotective effects of capsaicin and diallyl sulfide against mutagenesis or tumorigenesis by vinyl carbamate and $\mathrm{N}$-nitrosodimethylamine. Carcinogenesis. Oct 1995; 16(10): 2467-71.

[28] Henderson DE, Henderson SK. Thermal decomposition of capsaicin. 1. Interactions with oleic acid at high temperatures. J Agric Food Chem. Nov 1992; 40(11): 2263-8.
[29] Henderson DE, Slickman AM, Henderson SK. Quantitative HPLC determination of the antioxidant activity of capsaicin on the formation of lipid hydroperoxides of linoleic acid: A comparative study against BHT and melatonin. J Agric Food Chem. Jul 1999; 47(7): 2563-70.

[30] Morris VH, Cruwys SC, Kidd BL. Characterisation of capsaicin-induced mechanical hyperalgesia as a marker for altered nociceptive processing in patients with rheumatoid arthritis. Pain. Jun 1997; 71(2): 179-86.

[31] McElroy AP, Manning JG, Jaeger LA, Taub M, Williams JD, Hargis BM. Effect of prolonged administration of dietary capsaicin on broiler growth and Salmonella enteritidis susceptibility. Avian Dis. Abr-Jun 1994; 38(2): 329-33.

[32] Gutiérrez OL, Sumano LH, Zamora QM. Administration of enrofloxacin and capsaicin to chickens to achieve higher maximal serum concentrations. Vet Rec. Mar 2002; 150(11): 350-3. 